www.nature.com/pj

\title{
A time-of-flight study of a new hyperbranched oligomer film composed of thiophene and $N, N, N^{\prime}, N^{\prime}$-tetraphenyl-1,3-phenylenediamine
}

\author{
Masanobu Mizusaki ${ }^{1}$, Yuichiro Yamada ${ }^{1}$, Satoru Obara ${ }^{2}$, Kazushige Anzai ${ }^{2}$ and Kentaro Tada ${ }^{2}$
}

Polymer Journal (2011) 43, 431-434; doi:10.1038/pj.2011.8; published online 9 February 2011

Keywords: carrier transport property; soluble hyperbranched oligomer; thiophene; time-of-flight

\section{INTRODUCTION}

In recent years, much attention is being devoted to carrier transporting polymers with $\pi$-conjugation in both academic and industrial communities for their scientific and practical interest. ${ }^{1-5}$ In particular, $\pi$-conjugated soluble polymers have attracted considerable interest as potential candidates for use in light-emitting diodes, photovoltaic cells and field effect transistors, because they show unique features such as light-weight, flexibility and easy preparation in a large area. ${ }^{6-9}$ To achieve this, solubility is significantly important to prepare an uniform thin film without roughness and pinholes. ${ }^{5}$

For improving both solubility and carrier transport property, we were successful in designing $\pi$-conjugated soluble dendrimers, as reported in our earlier paper. ${ }^{10,11}$ The dendrimers are composed of $\pi$-conjugated $p$-phenylenevinylene dendrons surrounded by triphenylamine surface groups. ${ }^{10,11}$ The carrier mobility for the dendrimer film prepared by a spin-coating technique increased when the generation number of the $p$-phenylenevinylene group was decreased. ${ }^{11}$ The hole mobility for the film of a two-generation dendrimer attained an order of $10^{-4} \mathrm{~cm}^{2}(\mathrm{~V} \cdot \mathrm{s})^{-1}$ at $25^{\circ} \mathrm{C}$, whereas that of a three-generation dendrimer was in an order of $10^{-5} \mathrm{~cm}^{2}(\mathrm{~V} \cdot \mathrm{s})^{-1}$, as determined by a time-of-flight (TOF) method. ${ }^{11}$ Meier et al. ${ }^{12}$ reported that the molecular structure of the $p$-phenylenevinylene dendrimer surrounded by the alkyloxy groups becomes planar in shape with two-generation of $p$-phenylenevinylene. Thus, we can assume that the molecular structure of the two-generation dendrimer we prepared is planar in shape. ${ }^{11}$ In the case of the planar-shaped dendrimer, one can estimate that carrier hopping occurs easily as the distance between the $\pi$-conjugated groups of the different dendrimer molecules becomes comparably short. From the results of our earlier work, it can be anticipated that the $\pi$-conjugated soluble dendrimer is a candidate for practical use. However, a practical problem with the dendrimer is that the synthetic procedure is much complicated with a low yield, as compared with conventional polymers. This is particularly true for the thiophene dendrimer. ${ }^{13}$ Therefore, we are motivated to focus on the soluble $\pi$-conjugated hyperbranched oligomer, which can be expected to be comparably easily synthesized and useful for applications.

In this study, the photocarrier transport property of the film prepared by spin coating from solution of the newly synthesized soluble hyperbranched oligothiphene (see Figure 1) was investigated by the TOF method.

\section{EXPERIMENTAL PROCEDURE}

The chemical structure of the soluble hyperbranched oligothiophene ( $\mathrm{SH}$-oligothiophene), which was synthesized by our group, is shown in Figure 1. Bithiophene units are attached to a 1,3,5-phenylene core. Besides this, $N, N, N^{\prime}, N^{\prime}$-tetraphenyl-1,3-phenylenediamine groups are introduced to two of the bithiophene units for solubilization. The synthesized procedure was reported previously. ${ }^{14}$ The weight and number average molecular weights and the polydispersity index were $1.26 \times 10^{3}, 1.24 \times 10^{3}$ and 1.02 , respectively, ${ }^{14}$ as estimated by gel permeation chromatography with use of tetrahydrofuran as eluent and polystyrene standards for calibration.

A sandwich cell composed of indium tin oxide (ITO), SH-oligothiophene and an alloy of $\mathrm{Mg}$ and $\mathrm{Al}(\mathrm{Mg}-\mathrm{Al}), \mathrm{ITO} / \mathrm{SH}$-oligothiophene/Mg- $\mathrm{Al}$, was prepared as follows: first, a solution of SH-oligothiophene was prepared in chloroform at a concentration of $15 \mathrm{mg} \mathrm{ml}^{-1}$. The ITO substrate was masked, patterned and cleaned by sonication in $0.2 \mathrm{M}$ aqueous $\mathrm{NaOH}$ and deionized water. A SH-oligothiophene film was prepared from the chloroform solution onto the cleaned ITO substrate by spin coating. The thickness of the film was adjusted to $1.2 \mu \mathrm{m}$ by choosing a proper rotation speed. Next, a vacuum evaporator was used for the thermal evaporation of the alloy of $10 \mathrm{wt} \% \mathrm{Mg}$ and $90 \mathrm{wt} \% \mathrm{Al}(\mathrm{Mg}-\mathrm{Al})$ onto the desired ITO glass plate deposited with the SH-oligothiophene film.

TOF measurements for the sandwich cell of ITO/SH-oligothiophene/Mg-Al were carried out with a conventional setup, as described previously. ${ }^{15} \mathrm{~A}$ pulsed $\mathrm{N}_{2}$ laser ( $337.1 \mathrm{~nm}$, pulse width $0.7 \mathrm{~ns}$ ) having an output of $150 \mu \mathrm{J}$ per pulse was used to generate photocarrier in the SH-oligothiophene film. Measurements were recorded at $25^{\circ} \mathrm{C}$ under a vacuum condition $\left(10^{-3}\right.$ Torr $)$. The current generated as a result of photocarrier drift was collected across a $50 \Omega$ 


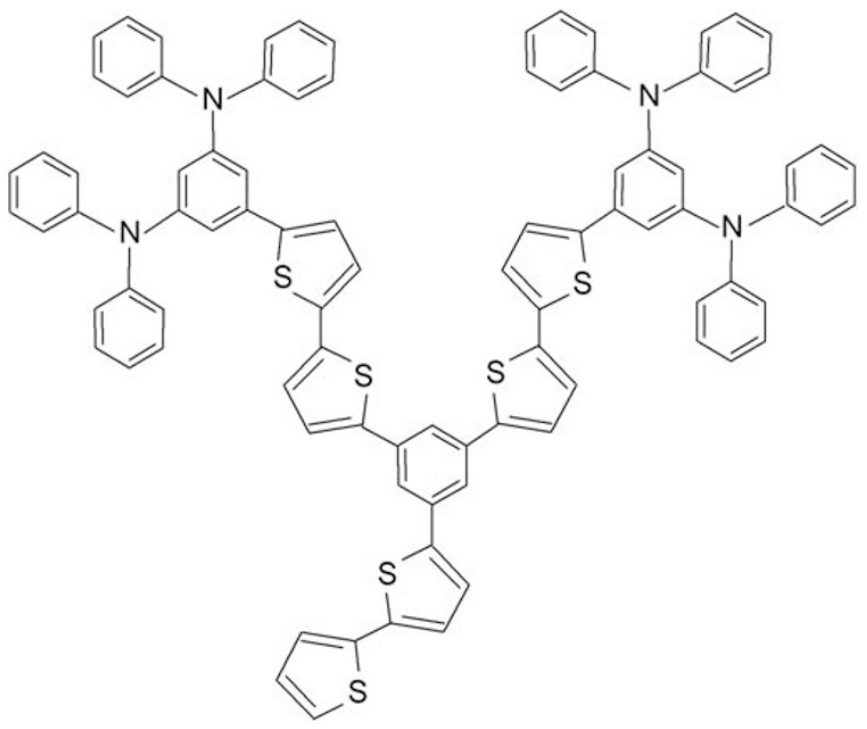

Figure 1 The soluble hyperbranched oligothiophene (SH-oligothiophene) used in this study.

resistor on application of an external bias voltage for a period of $120 \mu$ s. Carrier mobility was determined by the following equation

$$
\mu=\frac{L^{2}}{T_{\mathrm{tr}} V}
$$

where $\mu$ is the carrier mobility, $L$ is the thickness of the oligothiophene film, $V$ is the applied bias voltage and $T_{\text {tr }}$ is the transit time derived from the double logarithmic plot of the transient photocurrent. ${ }^{16}$

Absorption spectrum was measured on a Shimadzu UV-3100PC spectrophotometer (Shimadzu, Kyoto, Japan). For the measurement a 50-nm-thick SH-oligothiophene film prepared by spin coating from the chloroform solution on a glass substrate was used. Contribution from absorption of the glass substrate was corrected by subtracting the glass substrate spectrum from the absorption spectrum.

\section{RESULTS AND DISCUSSION}

The SH-oligothiophene dissolves in chloroform up to a concentration of $\sim 20 \mathrm{mg} \mathrm{ml}^{-1}$, which is more soluble than the dendrimers synthesized previously by us (that is, $\sim 10 \mathrm{mg} \mathrm{ml}^{-1}$ ). ${ }^{10,11}$ Shirota and Kageyama ${ }^{5}$ reported that the oligothiophenes are crystalline in nature. Hence, the solubility of the oligothiophenes is not enough to prepare films from the solutions. In this study, the introduction of the $N, N, N^{\prime}, N^{\prime}$-tetraphenyl-1,3-phenylenediamine group increases the solubility.

To ensure that photocarriers are generated by $\mathrm{N}_{2}$ laser irradiation for the TOF measurements, an absorption spectrum was measured. Figure 2 indicates the UV absorption spectrum for the SH-oligothiophene film of $50 \mathrm{~nm}$ thickness. Two typical absorption peaks centered at 300 and $380 \mathrm{~nm}$ are observed, the former due to the $N, N, N^{\prime}, N^{\prime}$ tetraphenyl-1,3-phenylenediamine group ${ }^{10}$ and the latter to the bithiophene group. It is obvious that the absorption bands for the $N, N, N^{\prime}, N^{\prime}$-tetraphenyl-1,3-phenylenediamine and bithiophene groups include the $337.1 \mathrm{~nm}$ wavelength of the $\mathrm{N}_{2}$ laser light, confirming that the photocarrier generation occurs by $\mathrm{N}_{2}$ laser irradiation.

By illuminating the $\mathrm{N}_{2}$ laser light from the ITO side of the ITO/SHoligothiophene/ $\mathrm{Mg}-\mathrm{Al}$ cell, photoexcited carriers of hole and electron generate at the surface of the SH-oligothiophene film. In cases in which a positive bias voltage is applied to the ITO side, the hole drifts from the ITO side to the $\mathrm{Mg}-\mathrm{Al}$ side and a positive photocurrent will be observed. On the other hand, the electron drifts and a negative

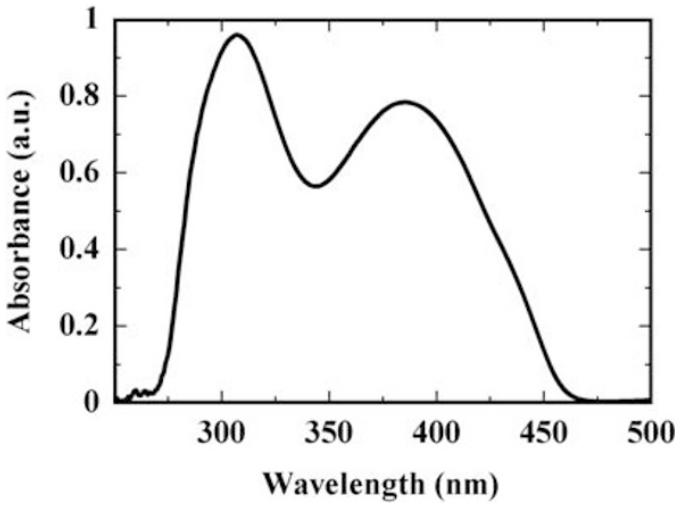

Figure 2 UV absorption spectrum for the SH-oligothiophene film.

photocurrent will be observed as a negative bias voltage is applied to the ITO side. Transient photocurrent profiles obtained from the ITO/SH-oligothiophene/ $\mathrm{Mg}-\mathrm{Al}$ cell are shown in Figures $3 \mathrm{a}$ and $\mathrm{b}$. Figure $3 \mathrm{a}$ indicates the transient photocurrent profiles at an applied voltage of +50 and $-50 \mathrm{~V}$ to the ITO side. When the positive bias voltage $(+50 \mathrm{~V})$ is applied to the ITO side, a much larger photocurrent is observed compared with the case in which the negative bias voltage $(-50 \mathrm{~V})$ is applied to the ITO side. This shows that the hole transport takes place preferentially compared with the electron transport. It is well known that both the thiophene and triphenylamine moieties show typical hole transport properties. ${ }^{5}$ Hence, the result is considered to be reasonable. Figure $3 \mathrm{~b}$ shows the transient photocurrent profiles in the range of the applied bias voltage from +50 to $+20 \mathrm{~V}$ to the ITO side. The photocurrent value decreases with decreasing applied bias voltage, indicating the possibility that the density of the hole transporting in the film decreases and the transport velocity of the hole becomes slow as the applied bias voltage decreases. All the transient photocurrent profiles shown in Figures $3 \mathrm{a}$ and $\mathrm{b}$ are typically dispersive, implying that the carrier transport property of the $\mathrm{SH}$ oligothiophene film is of a typical conducting polymer film type. ${ }^{5}$ As shown in the figures, dark currents seem to be small enough to be negligible, implying that the occurrence of carrier injection from the $\mathrm{Mg}-\mathrm{Al}$ electrode is negligible. Figure 4 shows the double logarithmic plots for the transient photocurrent profiles of hole in the range of the applied bias voltage from +50 to $+20 \mathrm{~V}$, which are modifications of Figure $3 \mathrm{~b}$. The transit time $\left(T_{\mathrm{tr}}\right)$, which is the intercept of two tangential lines before and after the inflection point, is observed in this range of the applied bias voltage. This is indicative of the fact that photocarriers travel across the SH-oligothiophene film and finally arrive to the $\mathrm{Mg}-\mathrm{Al}$ side at $T_{\mathrm{tr}} T_{\mathrm{tr}}$ qualitatively increases with decreasing applied bias voltage, confirming that the transport velocity of the hole becomes slow with decreasing applied bias voltage. As the

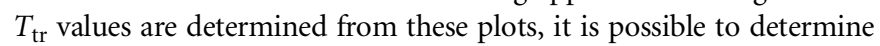
the mobility. ${ }^{16,17}$

Figures $5 \mathrm{a}$ and $\mathrm{b}$ show the current value at $T_{\mathrm{tr}}\left(I_{\mathrm{tr}}\right)$ and hole mobility derived from the double logarithmic plots for the transient photocurrent profiles as a function of applied bias voltage. $I_{\mathrm{tr}}$ increases with increasing applied bias voltage with a quadratic curve shown in Figure $5 \mathrm{a}$. Current value is represented with two factors of the transport velocity and the density of the carrier shown in equation (2).

$$
I \propto n \mu \frac{V}{L}
$$

where $I$ is the current value and $n$ is the density of the transporting carrier. In equation (2), $\mu(V / L)$ is the transport velocity and increases 

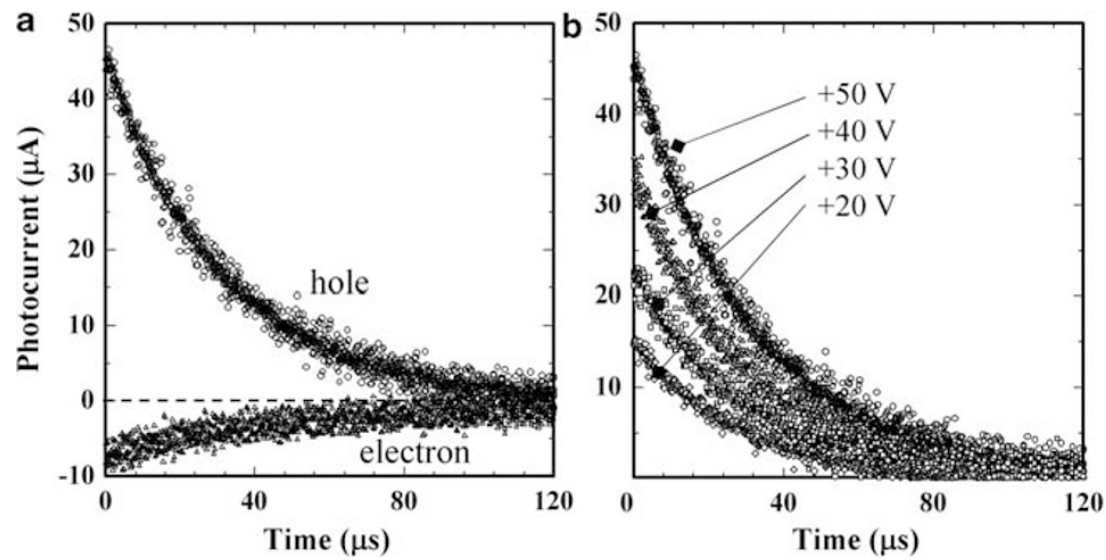

Figure 3 Profiles of transient photocurrents for (a) hole and electron transport at each applied bias voltage of +50 and $-50 \mathrm{~V}$, and for (b) hole transport at applied bias voltage from +50 to $+20 \mathrm{~V}$ of a ITO/SH-oligothiophene/Mg-Al cell.

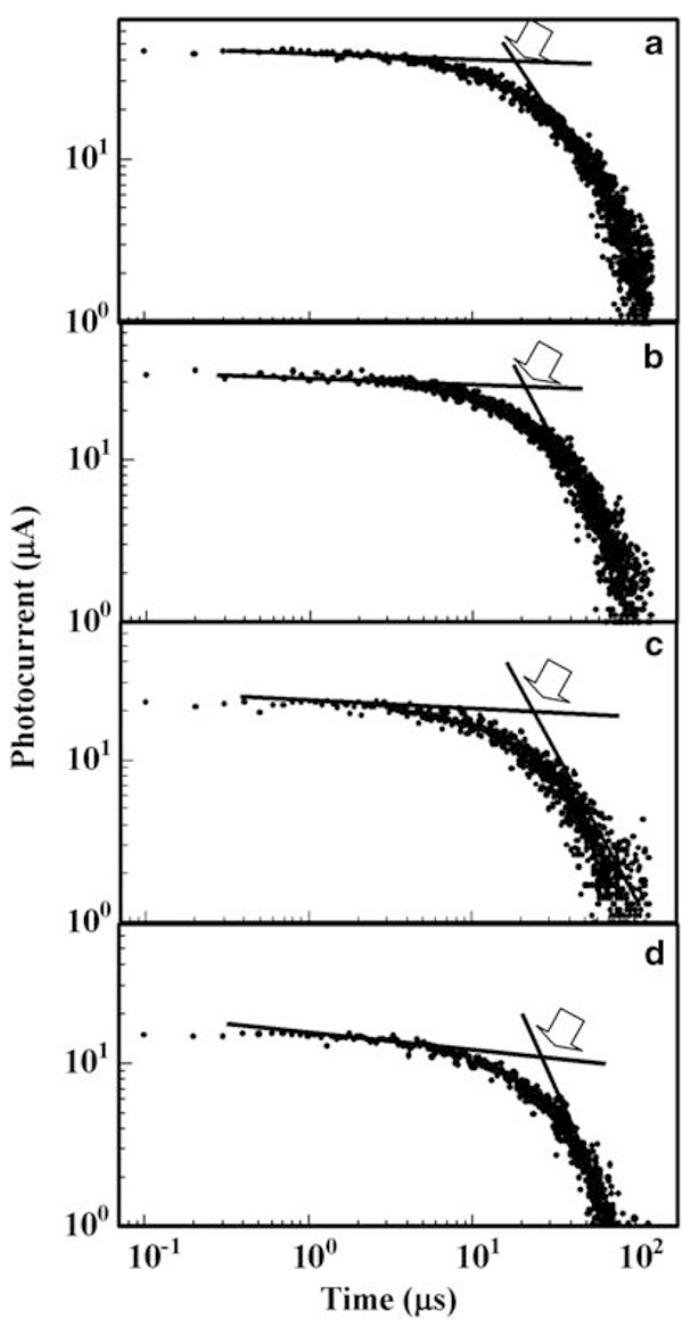

Figure 4 Profiles of double logarithmic transient photocurrents of the ITO/ $\mathrm{SH}$-oligothiophene/Mg-Al cell at applied bias voltage of (a) $+50 \mathrm{~V}$, (b) $+40 \mathrm{~V}$, (c) $+30 \mathrm{~V}$ and (d) $+20 \mathrm{~V}$.

linearly with increasing applied bias voltage. Thus, the quadratic curve in Figure $5 \mathrm{a}$ is considered to attribute to the increment of not only the transport velocity but also the density of the hole arrived to the $\mathrm{Mg}-\mathrm{Al}$

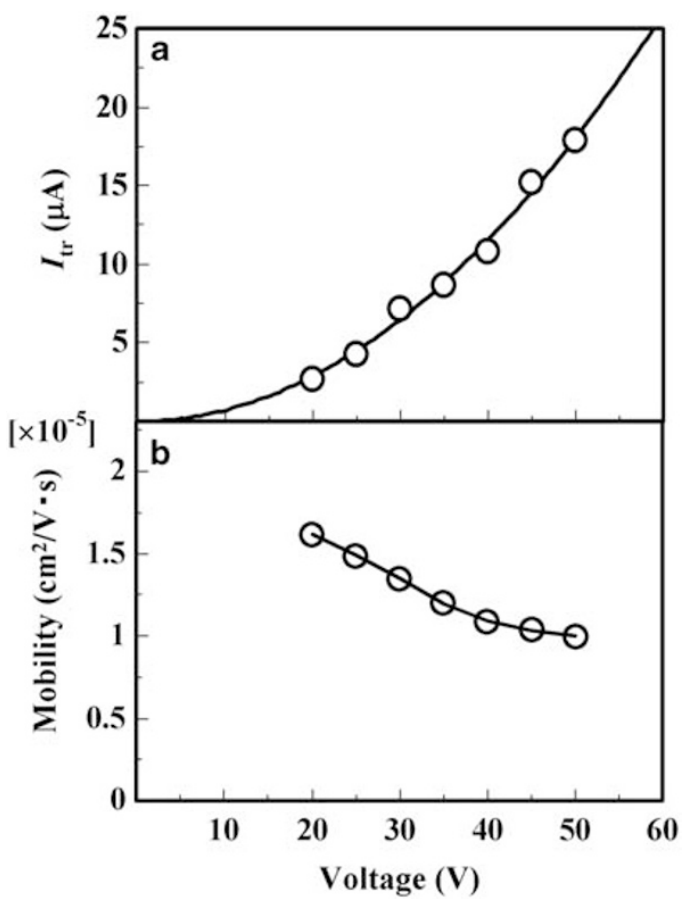

Figure 5 (a) Current at the transit time $\left(/_{\text {tr }}\right)$ and (b) hole mobility for the $\mathrm{SH}$ oligothiophene as a function of applied bias voltage.

side with increasing applied bias voltage. This tendency was observed in the two-generation p-phenylenevinylene dendrimer studied by us. ${ }^{11}$ As shown in Figure 5b, the hole mobility obtained by equation (1) ranges from $1.0 \times 10^{-5}$ to $1.6 \times 10^{-5} \mathrm{~cm}^{2}(\mathrm{~V} \cdot \mathrm{s})^{-1}$. It is to be noted that the mobility decreases with increasing applied bias voltage. As the dark currents are small enough to be negligible, this negative electric field dependence is explained by disorder formalism as follows. ${ }^{5}$ One can assume that the carrier transport in disordered systems takes place by hopping through many localized states subjected to the fluctuations of both hopping site energy and intermolecular wave function overlap, and that both the hopping site energy and the intermolecular distance follow the Gaussian distributions. The disorder is known to be shown for a variety of amorphous 
molecular glassy films. ${ }^{5}$ The hole mobility values of the SH-oligothiophene film are one order of magnitude smaller than those of the twogeneration dendrimer film evaluated previously by us. ${ }^{11}$ One of the causes is that the degree of disorder for the SH-oligothiophene film is higher than that for the two-generation dendrimer film. Here, the molecular weight distribution and diameter/height ratio of the two-generation dendrimer were 1.05 and 4.86 , respectively. ${ }^{11}$ A plausible reason for the high degree of disorder for the SH-oligothiophene film may be the flexible molecular structure of SH-oligothiophene, because the $\mathrm{SH}$-oligothiophene molecule has several single bonds that can rotate. In particular, the $N, N, N^{\prime}, N^{\prime}$-tetraphenyl-1,3-phenylenediamine group has seven single bonds between benzene and nitrogen groups or thiophene groups. When the thin films are prepared from solutions by spin coating, the control of molecular orientations is not easy. Thus, the controls of molecular structure and rigidity of the conducting molecules are estimated to be important for improvement of mobility.

\section{CONCLUSIONS}

The photocarrier transport property of the newly synthesized SHoligothiophene film prepared by spin coating from the solution was investigated by the TOF method. The SH-oligothiophene is dissolved in chloroform to obtain a concentration of $20 \mathrm{mg} \mathrm{m}^{-1}$, which is comparably of high solubility. The photocarrier of hole drifted in the SH-oligothiophene film preferentially, compared with electron, and the typical dispersive transport property was shown. The mobility of the hole derived from the transit time $\left(T_{\mathrm{tr}}\right)$ of the transient photocurrent profiles ranged from $1.0 \times 10^{-5}$ to $1.6 \times 10^{-5} \mathrm{~cm}^{2}(\mathrm{~V} \cdot \mathrm{s})^{-1}$ in the range of the applied bias voltage from +50 to $+20 \mathrm{~V}$. The mobility decreased with increasing applied bias voltage, being indicative of the disorder formalism of the SH-oligothiophene film. Further studies related to film morphology and molecular size are now being attempted.
1 Ito, T., Shirakawa, H. \& Ikeda, S. Thermal cis-trans isomerization and decomposition of polyacetylene. J. Polym. Sci. Polym. Chem. Ed. 13, 1943-1950 (1975).

2 Chiang, C. K., Gau, S. C., Fincher, C. R. Jr., Park, Y. W., MacDiarmid, A. G. \& Heeger, A. J. Polyacetylene, $(\mathrm{CH})_{x}: n$-type and $p$-type doping and compensation. Appl. Phys. Lett. 33, 18-20 (1978).

3 Dimitrakopoulos, C. D. \& Malenfant, P. R. L. Organic thin film transistors for large area electronics. Adv. Mater. 14, 99-117 (2002).

4 Chung, D. S., Lee, S. J., Park, J. W., Choi, D. B., Lee, D. H., Park, J. W., Shin, S. C., Kim, Y.- H., Kwon, S.- K. \& Park, C. E. High performance amorphous polymeric thinfilm transistors based on poly[(1,2-bis-(2'-thienyl)vinyl-5', $5^{\prime \prime}$-diyl)-alt-(9,9'-dioctylfluorene-2,7-diyl] semiconductors. Chem. Mater. 20, 3450-3456 (2008).

5 Shirota, Y. \& Kageyama, H. Charge carrier transporting molecular materials and their application in devices. Chem. Rev. 107, 953-1010 (2007).

6 Morrison, J. J., Murray, M. M., Li, X. C., Holmes, A. B., Morratti, S. C., Friend, R. H. \& Sirringhaus, $H$. Preparation of bis(dithienothiophene) derivatives for organic thin film transistors. Synth. Met. 102, 987-988 (1999).

7 Cravino, A., Roqet, S., Aleveque, O., Leriche, P., Frere, P. \& Roncali, J. Triphenylamineoligothiophene conjugated systems as organic semiconductors for opto-electronics. Chem. Mater. 18, 2584-2590 (2006).

8 Wang, Y., Zhou, E., Liu, Y., Xi, H., Ye, S., Wu, W., Guo, Y., Di, C., Sun, Y., Yu, G. \& Li, Y. Solution-processed organic field-effect transistors based on polythiophene derivatives with conjugated bridges as linking chains. Chem. Mater. 19, 3361-3363 (2007).

9 Wang, D., Kopidakis, N., Reese, M. O. \& Gregg, B. A. Treating poly(3-hexylthiophene) with dimethylsulfate improves its photoelectrical properties. Chem. Mater. 20, 6307-6309 (2008).

10 Mizusaki, M., Yamahara, M., Yamada, Y., Obara, S. \& Tada, K. Photocarrier transport property of phenylene-vinylene dendrimer studied by time-of-flight method. Polym. J. 41, 195-200 (2009).

11 Mizusaki, M., Yamahara, M., Yamada, Y., Obara, S. \& Tada, K. Carrier mobility for $\pi-$ conjugated dendrimer composed of phenylene-vinylene dendron and triphenylamine surface group. Polym. J. 41, 508-510 (2009).

12 Meier, H., Lehmann, M. \& Kolb, U. Stilbenoid dendrimers. Chem. Eur. J. 6, 2462 2469 (2000)

13 Xla, C., Fan, X., Locklin, J. \& Advincula, R. C. A first synthesis of thiophene dendrimers. Org. Lett. 4, 2067-2070 (2002).

14 Yamahara, M., Obara, S. \& Tada, K. Dendrimer and electronic device element employing the same. PCT Pub. No. WO2004/009679, 1-76 (2004).

15 Chen, B., Lee, C.- S., Lee, S.- T., Webb, P., Chan, Y.- C., Gambling, W., Tian, H. \& Zhu, W. Improved time-of flight technique for measuring carrier mobility in thin films of organic electroluminescent materials. Jpn. J. Appl. Phys. Part 1 39, 1190-1192 (2000).

16 Shcer, H. \& Montroll, E. W. Anomalous transit-time dispersion in amorphous solids. Phys. Rev. B 12, 2455-2477 (1975).

17 Lee, S. H., Yasuda, T. \& Tsutsui, T. Charge carrier mobility in blue-green emitting fluorenylsubstituted poly(p-phenylene vinylene)s. J. Appl. Phys. 95, 3825-3827 (2004). 